\title{
Nématodes Héligmosomes
}

\section{parasites d'Insectivores Talpidés \\ de la région holarctique}

\author{
par Marie-Claude DURETTE-DESSET et Claude VAUCHER \\ Laboratoire de Zoologie (Vers), associé au C.N.R.S. ( $\mathrm{P}^{\mathrm{r}}$ A.-G. Chabaud), \\ Muséum national d'Histoire naturelle, 43, rue Cuvier, F 75231 Paris Cedex 05 \\ et Institut de Zoologie ( $\mathrm{P}^{\mathrm{r}}$ J.-G. BAER), Université de Neuchâtel, \\ rue Emile-Argand, 2000 Neuchâtel 7, Suisse
}

\section{Résumé}

Dans ce travail, nous étudions le synlophe de deux espèces: Tricholinstowia linstowi (Travassos, 1918) et $T$. talpae (Morgan, 1928). Deux nouvelles espèces sont décrites: $T$. mornanti n. sp., parasite de Talpa europea en France, caractérisée par ses spicules courts, mais à pointes simples, et $T$. maseri n. sp., parasite de Scapanus orarius aux U.S.A., caractérisée par son nombre élevé d'arêtes cuticulaires.

Nous avons essayé de sérier les espèces en tenant compte de l'évolution de trois caractères: les spicules, la côte dorsale et le synlophe.

Il n'y a pas de corrélation nette entre ces trois caractères, cependant la nouvelle espèce néarctique $T$. maseri apparaît nettement plus évoluée que les autres espèces du genre.

\section{Summary}

Nematodes Heligmosomes parasites of Insectivora Talpidae from holarctic region.

In this work, the synlophe of two species: Tricholinstowia linstowi (Travassos, 1918) and T. talpae (Morgan, 1928) are studied. Two new species are described: T. mornanti n. sp., parasite of Talpa europea from France is characterized by short and single tipped spicules. T. maseri n. sp. parasite of Scapanus orarius from the United States, which is differentiated by its large number of cuticular ridges. 
An attempt has been made to classify the species on account of the three main following evolutive characters : i.e. spicules, dorsal ridges, and synlophe.

There is no well pronounced relationship between these three features; nevertheless the new nearctic species $T$. maseri has achieved a more advanced stage of evolution than the other species of the genus do.

\section{INTRODUCTION}

Les Nématodes Héligmosomes parasites d'Insectivores Talpidés décrits jusqu'à présent appartiennent au genre Tricholinstowia Travassos, 1937 (1) et ne sont connus que de la région paléarctique.

Grâce à du matériel de France aimablement communiqué par nos amis $\mathbf{M}$. et $\mathbf{M}^{\mathrm{me}}$ Mornant, et M. J.-Cl. Quentin, nous avons pu faire une étude du synlophe de ce genre.

D'autre part, nous devons à l'obligeance du $\mathrm{D}^{\mathrm{r}}$ R.-L. Rausch l'envoi de matériel récolté chez des Scapanus américains par le $\mathrm{D}^{r} \mathrm{C}$. Maser (2). Cette espèce est nouvelle et élargit la répartition du genre Tricholinstowia à la région néarctique.

Tous les spécimens étudiés sont déposés dans les collections du Muséum national d'Histoire naturelle de Paris.

\section{DESCRIPTION DES ESPECES}

\section{- Tricholinstowia linstowi (Travassos, 1918)}

MatérIel : 1 đ̊ trouvé dans la moitié postérieure de l'intestin grêle avec de nombreux stades larvaires (tube $866 \mathrm{M}$ ).

Hôte : Talpa europea ?. L.

Origine GÉOGRAPHIQUe : Nangis (France).

Synlophe: Corps parcouru longitudinalement par 9 arêtes cuticulaires dont 3 latérales droites et 6 ventrales gauches. En coupe transversale, la pointe des arêtes est dirigée de la ligne ventrale-droite vers la ligne dorsale-gauche pour les deux faces avec un gradient de taille de droite à gauche pour la face latérale droite et de gauche à droite pour la face ventrale. La face dorsale est presque entièrement inerme, mais la cuticule est dentelée (fig. 1, A, B, C).

(1) Le genre Morganiella Travassos 1937 étant préemployé, Fahmy en 1956 l'a remplacé par le taxon Morganostrongylus. L'espèce type de ce genre, $M$. talpae, étant pour nous un Tricholinstowia, le genre Morganostrongylus tombe en synonymie de Tricholinstowia Travassos 1937.

(2) Nous remercions vivement les $\mathrm{D}^{\mathrm{rs}}$ R.-L. Rausch et Chris Maser pour ce matériel qui fait partie d'un envoi important non encore étudié. 

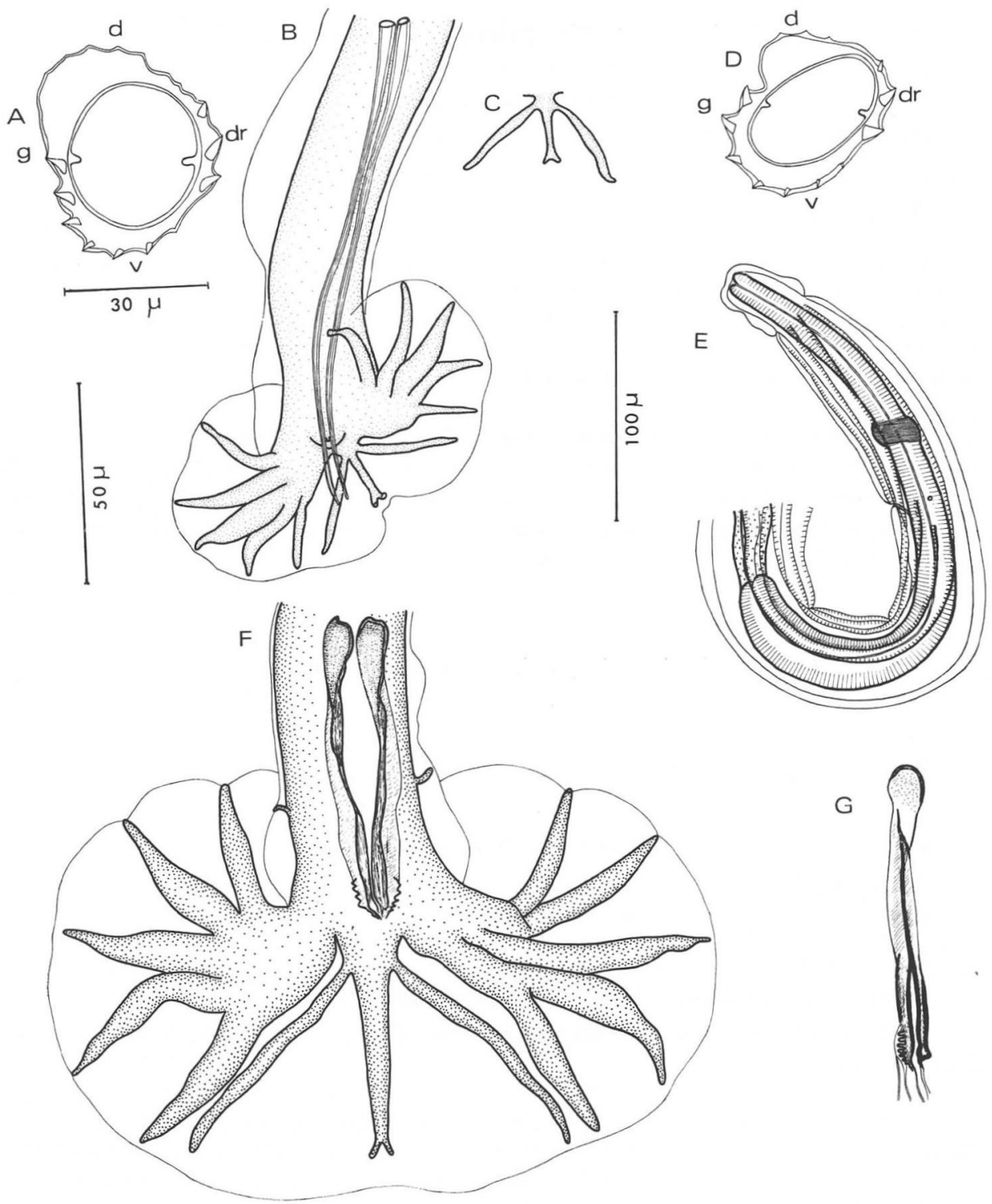

FIG. 1. - Tricholinstowia linstowi (Travassos, 1918), ô. A, coupe transversale au milieu du corps.

B, bourse caudale, vue ventrale. C, détail des côtes 8-9-10, vue dorsale. - T. talpae (Morgan, 1928), 3. D, coupe transversale au milieu du corps. E, naissance des arêtes cuticulaires, extrémité antérieure, vue latérale gauche. $\mathrm{F}$, bourse caudale, vue ventrale. $\mathrm{G}$, spicule disséqué. A, éch. $0,03 \mathrm{~mm}$. B, C, D, F, G, éch. $0,05 \mathrm{~mm}$. E, éch. $0,1 \mathrm{~mm}$. 


\section{- Tricbolinstowia talpae (Morgan, 1928)}

MAtÉRIEL : 2 o, 1 en mauvais état (tube $255 \mathrm{M}$ ).

Hôte : Talpa europea $\delta$ L.

Localisation : Duodenum.

Origine GÉOGRAPHIQUe: Nangis (France).

Synlophe: Corps parcouru longitudinalement par 9 arêtes cuticulaires, 3 latérales droites et 6 ventrales gauches. En coupe transversale, la pointe des arêtes est dirigée de la ligne ventrale-droite vers la ligne dorsale-gauche. Gradient de taille de droite à gauche pour la face latérale droite et de gauche à droite pour la face ventrale. Face dorsale presque entièrement inerme, mais avec la cuticule dentelée (fig. 1, D à G).

Discussion: Malgré ses spicules courts et complexes, nous avons classé cette espèce dans le genre Tricholinstowia Travassos, 1937 (cf. Durette-Desset, 1971), car son synlophe est identique à celui de l'espèce type. Nous devons noter également la grande similitude entre les bourses caudales, et en particulier la disposition des côtes 5 et 6 formant "pince» (voir fig. 1, B et F).

\section{- Tricholinstowia mornanti n. sp.}

MAtÉRIEl TYPE : 10 *, 10 (tube $223 \mathrm{KA}$ ).

Hôte : Talpa europea L. đ.

LOCALISATION : intestin grêle.

Origine GÉOGRAPHIQUe: Coudray-au-Perche, Orne (France).

Autre matériel : 5 ô (tube $254 \mathrm{M}$ ).

HôTe: Talpa europea L. + .

LOCALISATION : intestin grêle.

Origine GÉOgraphiQue: Nangis, Seine-et-Marne (France).

Description : Très petits Nématodes enroulés de façon assez lâche le long de leur ligne ventrale.

Synlophe: Chez les 2 sexes, le corps est parcouru longitudinalement par 9 arêtes cuticulaires, dont 3 latérales droites et 6 ventrales gauches. En coupe transversale, la pointe des arêtes est dirigée de la ligne ventrale-droite vers la ligne dorsale-gauche. Le gradient de taille des arêtes est de gauche à droite sur la face ventrale et de droite à gauche sur la face dorsale.

Les arêtes naissent pour la plupart sur le bord postérieur de la vésicule céphalique et quelques-unes sur le champ latéral gauche.

Dans la seconde moitié du corps, elles sont beaucoup moins marquées et elles disparaissent à environ $500 \mu$ de la pointe caudale ou de la bourse caudale (fig. 2, B). 


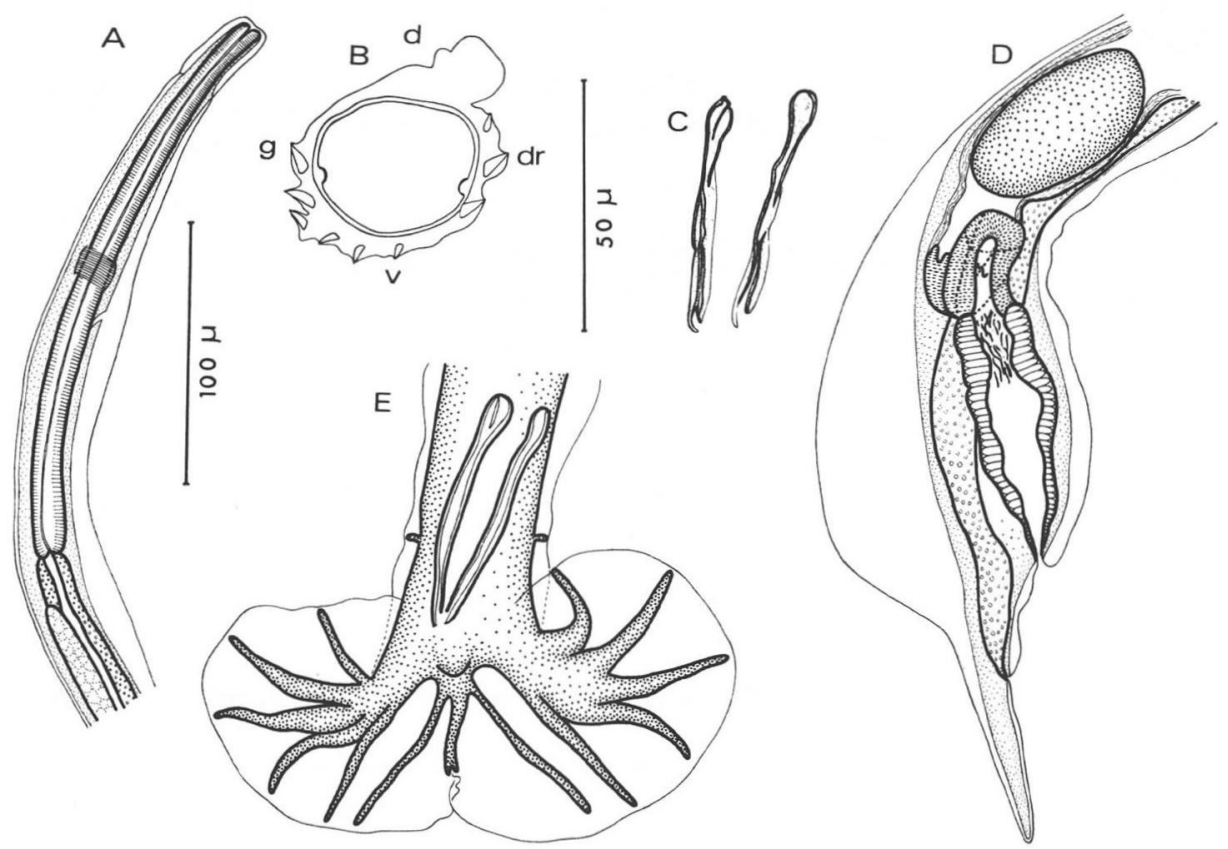

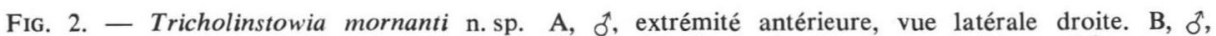
coupe transversale au milieu du corps. C, $\delta$, spicules disséqués. D, $q$, extrémité postérieure, vue latérale droite. $\mathrm{E}$, $\delta$, bourse caudale, vue ventrale.

A, D, éch. $0,1 \mathrm{~mm}$. B, C, E, éch. $0,05 \mathrm{~mm}$.

Mâle : corps long de $1,17 \mathrm{~mm}$, large de $50 \mu$ dans sa partie moyenne. Vésicule céphalique haute de $40 \mu$ sur $20 \mu$ de large. Anneau nerveux et pore excréteur situés respectivement à $110 \mu$ et $130 \mu$ de l'apex. Deirides non vues. CEsophage long de $240 \mu$ (fig. 2, A).

Bourse caudale sub-symétrique figurée en 2, E. Papilles prébursales présentes. Extrémité des côtes 5 dirigée vers les côtes 6 . Côtes 8 grêles plus longues que la côte dorsale. Cette dernière est divisée en deux petits rameaux à son extrémité. Gubernaculum absent.

Spicules courts, relativement complexes, ailés, longs de $47 \mu$. Leur extrémité distale est dépourvue d'indentations (fig. 2, C).

Femelle: Corps long de $1,9 \mathrm{~mm}$, large de $60 \mu$ dans sa partie moyenne. Vésicule céphalique haute de $40 \mu$ sur $25 \mu$ de large. Anneau nerveux et pore excréteur situés respectivement à $105 \mu$. et $120 \mu$ de l'apex. Deirides non vues. Esophage long de $220 \mu$.

Appareil génital monodelphe. La vulve s'ouvre à $110 \mu$ de la pointe caudale. Vestibule, sphincter et trompe longs respectivement de $62 \mu$, $28 \mu$ et $70 \mu$. L'utérus mesure $310 \mu$ et contient trois œufs non embryonnés hauts de $75 \mu$ sur $40 \mu$ de large (fig. 2, D).

Queue effilée, longue de $65 \mu$.

Discussion: Cette espèce présente les principaux caractères du genre Tricholinstowia, en particulier le synlophe et la morphologie bursale. Elle se distingue cepen- 
dant des autres espèces du genre par ses spicules courts dont les extrémités distales sont bifides et arrondies.

Nous pensons donc que cette espèce est nouvelle, et nous la nommons Tricholinstowia mornanti n. sp., en la dédiant à M. P. Mornant.

\section{- Tricholinstowia maseri n. sp.}

MATÉRIEL TYPE : nombreux ô et $q$ (1001 KA).

Hôte : Scapanus orarius $\delta$ True.

LOCALISATION : intestin grêle.

Origine GÉOgraphiQue: Tahkenitch Lake, Oregon (U.S.A.).

Autre matériel : 5 ô, 24 (1002 KA).

Hôte: Scapanus orarius ô True.

LOCALISATION : intestin grêle.

ORIGINE GÉOGRAPHIQUe : 9 miles au nord de Gardiner, Lane Co, Oregon (U.S.A.).

Description: Petits Nématodes enroulés de façon senestre le long de la ligne ventrale. Les tours de spire sont fortement serrés dans la partie antérieure du corps.

Synlophe: Chez les deux sexes, le corps est parcouru longitudinalement par six arêtes latéro-dorsales droites et dix arêtes ventrales. En coupe transversale, leur pointe apparaît dirigée de la ligne ventrale-droite vers la gauche. Face latéro-dorsale gauche inerme. Gradient de taille de gauche à droite pour les arêtes ventrales et de droite à gauche pour les arêtes latéro-dorsales droites. Les deux arêtes droites sont les plus fortes (fig. 3, C). La plupart des arêtes naissent sur le bord postérieur de la vésicule céphalique et les autres s'étagent sur le champ latéral gauche (fig. 3, B). Fortement marquées dans la partie antérieure du corps, leur taille diminue progressivement et elles disparaissent à peu près aux $4 / 5$ de la longueur du corps. La cuticule est fortement dilatée, surtout sur la face ventrale.

Mâle : Corps long de $1,85 \mathrm{~mm}$ sur $75 \mu$ de large. Vésicule céphalique haute de $45 \mu$ sur $20 \mu$ de large. Anneau nerveux, pore excréteur et déirides situés respectivement à $140 \mu$, $185 \mu$ et $185 \mu$ de l'apex. Esophage long de $380 \mu$ (fig. 3, A).

Bourse caudale sub-symétrique. Papilles prébursales présentes. Côtes 4 épaisses et plus longues que les 5. Tronc commun aux côtes 5 et 6 , celles-ci ont une disposition caractéristique figurée en 3 , G. Côtes 8 très épaisses, naissant assez haut sur la côte dorsale. Cette dernière se divise à son extrémité en quatre rameaux. Les deux médians sont les plus courts (fig. 3, G).

Spicules ailés, longs de $115 \mu$, à extrémité distale bifide (fig. 3, F). Absence de gubernaculum. Cône génital triangulaire, fortement chitinisé (fig. 3, G).

Femelle: Corps long de $2,8 \mathrm{~mm}$, large de $75 \mu$. Vésicule céphalique haute de $50 \mu$ sur $22 \mu$. de large. Anneau nerveux et pore excréteur situés respectivement à $140 \mu$ et $170 \mu$. Deirides non vues. Esophage long de $380 \mu$. 


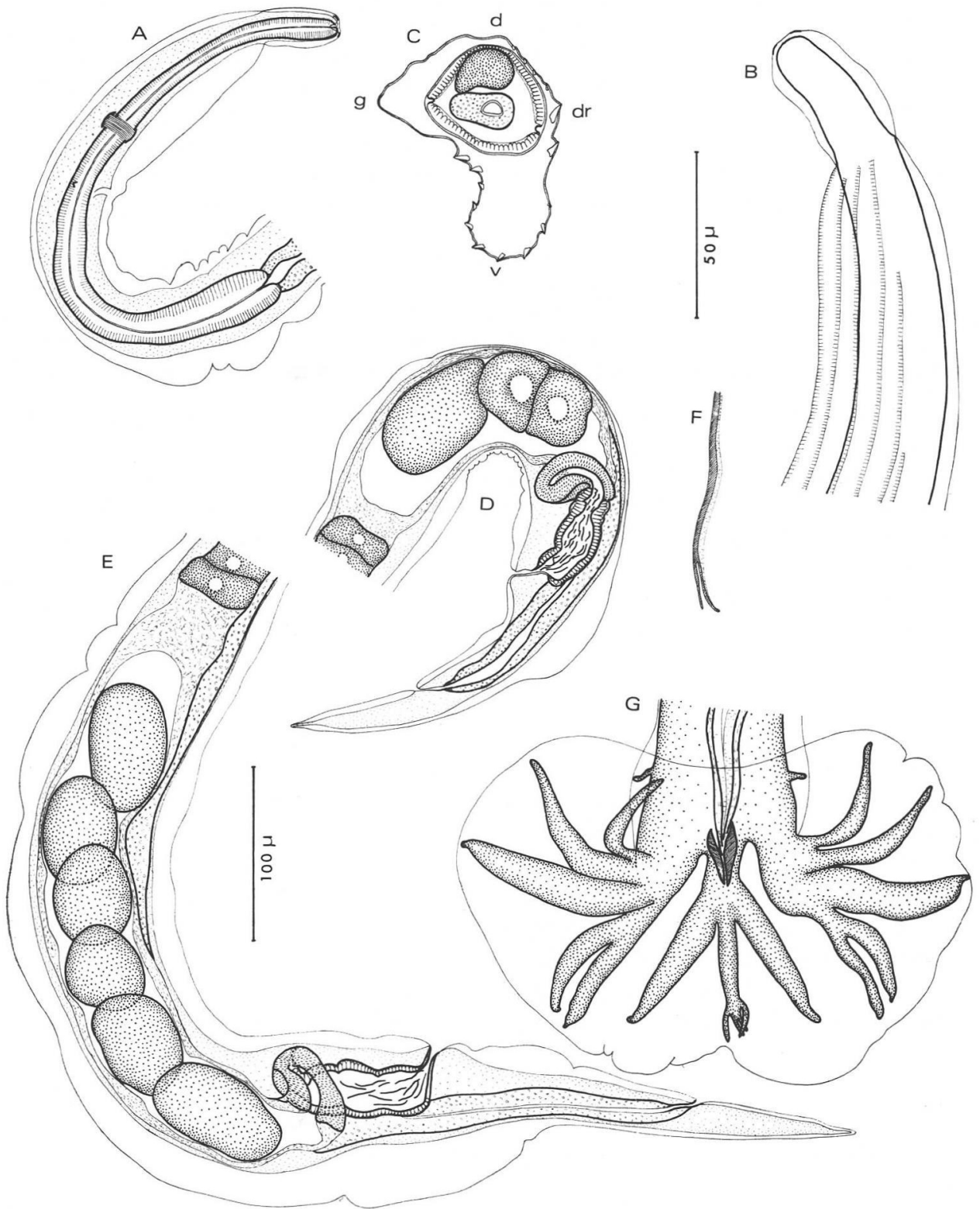

FIG. 3. - Tricholinstowia maseri n. sp. A, ơ, extrémité antérieure, vue latérale droite. B, idem, naissance des arêtes cuticulaires, vue latérale gauche. $\mathrm{C}, q$, coupe transversale au milieu du corps.

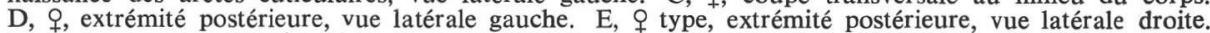
$\mathrm{F}, \hat{\delta}$, pointe d'un spicule. $\mathrm{G}, \hat{\circ}$, bourse caudale, vue ventrale. A, D, E, éch. 0,1 mm. B, C, F, G, éch. 0,05 mm. 
Appareil génital monodelphe. La vulve s'ouvre à $250 \mu$ de la pointe caudale. Ovéjecteur assez court de $125 \mu$ de long, avec un vestibule, un sphincter et une trompe, longs respectivement de $50 \mu, 15 \mu$. et $60 \mu$. Utérus long de $360 \mu$, contenant six œufs non embryonnés, hauts de $75 \mu$ sur $42 \mu$ de large. L'ovaire débute en arrière de la jonction œsophage-intestin.

La queue est effilée, longue de $92 \mu$ (fig. 3, E).

Variations: Environ le tiers des spécimens du tube $1001 \mathrm{KA}$ et la grande majorité du tube $1002 \mathrm{KA}$ sont nettement plus petits que les spécimens décrits ci-dessus.

Chez les mâles, la morphologie des bourses caudales est strictement identique à celle des grands spécimens. Les spicules sont plus courts $(110 \mu$ pour un mâle de $1,3 \mathrm{~mm})$.

Chez les femelles, la différence de taille n'affecte pas l'ovéjecteur, mais la longueur de l'utérus : chez une femelle de $1,8 \mathrm{~mm}$, l'ovéjecteur mesure $120 \mu$ et l'utérus $165 \mu$ (fig. 3, D). Cette femelle était mûre, car elle contenait deux œufs, dont un au stade 2 blastomères.

La présence de grands et de petits spécimens serait peut-être dûe à deux infestations successives, étant donné que nous n'avons pas trouvé de spécimens de taille intermédiaire.

Discussion : Les spécimens répondent à notre nouvelle définition du genre Tricholinstowia (cf. Durette-Desset, 1971).

Ils se distinguent immédiatement des espèces dont le synlophe est connu par le nombre plus élevé des arêtes cuticulaires. La forme de la pointe des spicules, l'épaisseur des côtes 8 et le rapprochement des côtes 5 et 6 les séparent des autres espèces du genre.

Nous pensons donc que les spécimens du Scapanus sont nouveaux et nous les dédions à M. Chris Maser, en les nommant Tricholinstowia maseri $\mathrm{n}$. sp.

\section{CONCLUSION}

Nous connaissons maintenant sept espèces de Tricholinstowia, dont six dans la région paléarctique et une dans la région néarctique.

Les caractères morphologiques sont très proches d'une espèce à l'autre, en particuilier le synlophe et la morphologie bursale.

Nous avons tenté de juger du degré d'évolution de chaque espèce en tenant compte de trois caractères: les spicules, la côte dorsale, le synlophe.

1) Les spicules. L'évolution s'effectue par un allongement de leur taille et une simplification de leur extrémité distale.

a) Les spicules sont courts et à pointe complexe chez $T$. talpae et $T$. mornanti, courts mais à pointe simple chez T. mogera (Sadovskaja, 1952) et T. morenishi (Cameron et Parnell, 1933).

b) Les spicules sont longs et à pointes simples chez T. vigisi (Petrow et Savinow, 1959), T. maseri et T. linstowi.

2) La côte dorsale. L'évolution s'effectue par une réduction de la longueur de cette côte (cf. Chabaud, 1959).

a) La côte dorsale est plus longue que les côtes 8 chez T. mogera et $T$. vigisi.

b) La côte dorsale est aussi longue que les côtes $8 \mathrm{chez} T$. talpae, T. morenishi et $T$. maseri.

c) La côte dorsale est plus courte que les côtes 8 chez $T$. linstowi et $T$. mornanti. 
3) Le synlophe. L'évolution s'effectue par une augmentation du nombre des arêtes cuticulaires. Les espèces paléarctiques ont exactement le même synlophe, avec 9 arêtes (1). Par contre, l'espèce néarctique $T$. maseri a un synlophe nettement plus évolué, avec 16 arêtes.

Nous pouvons donc classer les espèces en trois groupes :

- Groupe A:T. talpae, T. mornanti; les spicules restent primitifs, mais il y a réduction de la côte dorsale.

- Groupe B: T. mogera, T. vigisi; la côte dorsale reste primitive, mais les spicules s'allongent.

- Groupe $C: T$. morenishi, T. linstowi ; les spicules s'allongent et la côte dorsale se réduit.

Enfin, l'espèce $T$. maseri peut être rattachée au groupe $\mathrm{B}$, en ce qui concerne les caractères de la dorsale et des spicules, mais elle est nettement séparée des autres espèces par son synlophe plus évolué.

Nous avons schématisé ces éléments sur la figure 4.

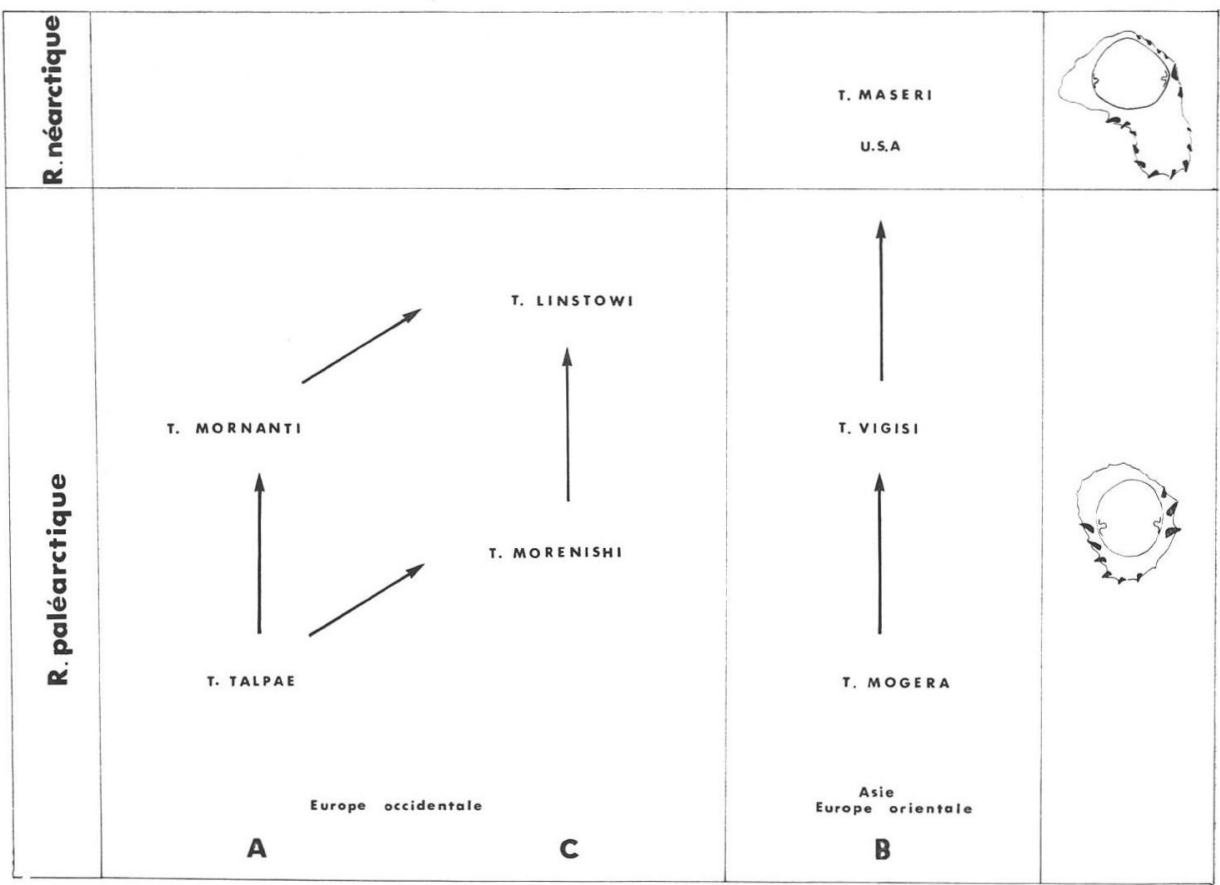

FIg. 4. - Essai de classification des espèces du genre Tricholinstowia Travassos 1937 en function de l'évolution de trois caractères : les spicules, la côte dorsale et le synlophe.

(1) Ce synlophe n'est connu que chez trois espèces sur six. 


\section{Bibliographie}

Cameron (T. W. M.) et Parnell (I. W.), 1933. - The internal parasites of land mammals in Scotland. Proc. R. Phys. Soc., 22, 133-154.

Durette-Desset (M.-C.), 1971. - Essai de classification des Nématodes Héligmosomes. Corrélations avec la paléobiogéographie des hôtes. Mém. Mus. Nat. Hist. Nat., sér. A. Zool., 69, 126 pp.

FAHMY (M. A. M.), 1956. - Studies on Morganiella talpae and the taxonomic consideration of the genus Morganiella (Nematode, Trichostrongylidae). Z.f. Parasitenk., 17, 346-348.

Morgan (D. O.), 1928. - A new nematode species of the genus Viannaia from the mole (Talpa europea). J. Helminth., 6, 199-204.

Petrow (A. M.) et Savinow (V. A.), 1959. - «On helminth fauna of moles (Talpa europaea) in the Kalinin region 》 Russian text. Trudy Vses. Inst. Gel'mint, 6, 160-166.

SAdovsKaja (N. P.), 1952. - Vers parasites de Rongeurs et Insectivores du littoral. Compte rendu de dissertation cité par Skrjabin, Schikhobalowa et Schulz, 1954.

Skrjabin (K. I.), Schikhobalowa (N. P.) et Schulz (R. S.), 1954. - Osnovi Nematodologi IV, Dictyocaulides et Heligmosomatides, $323 \mathrm{pp}$.

Travassos (L.), 1920. - Trichostrongylidae brasileiros. Rev. Soc. Brasil, Sc., (1919), 3, 191205.

—, 1937. - Revisao da familia Trichostrongylidae Leiper, 1912. Monogr. Inst. O. Cruz. I, $512 \mathrm{pp}$. 